\title{
Proton induced reactions on the p-nucleus ${ }^{120} \mathrm{Te}$
}

\author{
N. Özkan ${ }^{1}$, R. T. Güray, C. Yalçın, S. Kutlu
}

Kocaeli University

Department of Physics, TR-41380 Umuttepe, Kocaeli, Turkey

\section{A. Palumbo, R. deBoer, J. Görres, P. J. Leblanc, S. O'Brien, E. Strandberg,}

\section{W. P. Tan, M. Wiescher}

University of Notre Dame

Notre Dame, Indiana 46556, USA

\section{H. Y. Lee}

Argonne National Laboratory

Argonne, IL 60439 USA

\section{Zs. Fülöp, E. Somorjai}

Institute of Nuclear Research (ATOMKI)

H-4001 Debrecen, POB. 51., Hungary

In order to extend the experimental database related to the p-process, the ${ }^{120} \mathrm{Te}(\mathrm{p}, \gamma){ }^{121} \mathrm{I}$ reaction cross section has been measured with high precision by means of the activation method in the effective center of mass energies between $2.47 \mathrm{MeV} \leq \mathrm{E}_{\mathrm{c} . \mathrm{m} .}^{\text {eff }} \leq 7.93 \mathrm{MeV}$. The covered energy range spans the Gamow window predicted in the high temperature environment, e.g., $2.43 \mathrm{MeV} \leq \mathrm{E}_{\mathrm{G}} \leq$ 4.64 MeV for $\mathrm{T}_{9}=3$. In addition, the ${ }^{120} \mathrm{Te}(\mathrm{p}, \mathrm{n})^{120} \mathrm{I}$ reaction cross section has been investigated in the energy range $6.44 \mathrm{MeV} \leq \mathrm{E}_{\mathrm{c.m} .}^{\mathrm{eff}} \leq 7.93 \mathrm{MeV}$. The targets were prepared by evaporation of $99.4 \%$ isotopically enriched ${ }^{120} \mathrm{Te}$ on Aluminum and Carbon backing foils, and bombarded with proton beams provided by the FN Tandem Van de Graaff accelerator at the University of Notre Dame. The cross sections were deduced from the observed induced $\gamma$ ray activity, which was detected off-line by two Clover HPGe detectors placed face to face in close geometry. The cross section measurements were also compared with the predictions of statistical model calculations using NONSMOKER code to test the reliability of the theoretical input parameters.

10th Symposium on Nuclei in the Cosmos

Mackinac Island, Michigan, USA

27 July - 1 August, 2008

\footnotetext{
${ }^{1}$ Speaker, E-mail: nozkan@kocaeli.edu.tr
} 


\section{Introduction}

The p-process is the least known among three main nucleosynthesis processes: s-process, r-process, and p-process. The p-process can proceed via a combination of photodisintegration reactions, $(\gamma, n),(\gamma, p)$ and $(\gamma, \alpha)$ on existing heavy s- and r-seeds in the temperature range of $2-$ $3 \times 10^{9} \mathrm{~K}$ [1], [2]. Since most $\gamma$-induced reactions [3], [4] are difficult to measure directly, the photon-induced reaction cross sections can be determined by measuring the inverse reactions using the activation method, if the product is radioactive and has an appropriate $\beta$-decay halflife with an adequate $\gamma$-intensity. The obtained results are converted to $\gamma$-induced reaction cross sections by using the detailed balance theorem. In the last decade, the interest in measuring the cross sections of charged particle induced reactions has increased [5-17], see Ref. [18] for the detail. Although the $(p, \gamma)$ measurements generally agree with the statistical model predictions within less than a factor of two, more (p, $\gamma$ ) data in the relevant energy range will be useful for a direct application in reaction network. Meantime, for a comparison, (p,n) measurements are not available at all except for ${ }^{76} \mathrm{Ge}$ [5] and ${ }^{82} \mathrm{Se}$ [6] isotopes. Moreover, experimental data for the charged particle induced reaction cross sections are scarce for higher mass region, especially $A>100$. It is, therefore, important to investigate experimentally the p-induced reaction cross sections on ${ }^{120} \mathrm{Te}$, which is a p-nucleus, in order to test the reliability of the statistical model predictions.

This work presents the measurements of ${ }^{120} \mathrm{Te}(\mathrm{p}, \gamma){ }^{121} \mathrm{I}$ and ${ }^{120} \mathrm{Te}(\mathrm{p}, \mathrm{n}){ }^{120} \mathrm{I}$ reaction cross sections in the astrophysical energy range (the energy range for Gamow window is between 2.43 $\mathrm{MeV}$ and $4.64 \mathrm{MeV}$ at the temperature of $3 \times 10^{9} \mathrm{~K}$ ).

\section{Experimental method}

Measurements of (p, $\gamma)$ and $(p, n)$ cross sections on targets of ${ }^{120}$ Te were carried out in a proton beam energy range from $2.5 \mathrm{MeV}$ to $8.0 \mathrm{MeV}$ in the laboratory frame with steps of 0.5 $\mathrm{MeV}$, corresponding to effective center of mass energies from $2.47 \mathrm{MeV}$ to $7.93 \mathrm{MeV}$. As the beam defining aperture, a $10 \mathrm{~mm}$ diameter collimator was placed just before the target irradiation chamber. A secondary electron suppression voltage of $-300 \mathrm{~V}$ was also applied. Total number of incident protons is measured with a Faraday cup mounted right after the target chamber. Enriched ${ }^{120} \mathrm{Te}$ oxide (99.4\%) material was evaporated onto $20 \mu \mathrm{g} . \mathrm{cm}^{-2} \mathrm{C}$ [19] and $1500 \mu \mathrm{g} . \mathrm{cm}^{-2} \mathrm{Al}$ backing, and two targets were provided with the thickness of $128 \mu \mathrm{g} . \mathrm{cm}^{-2}$ and $456 \mu \mathrm{g} . \mathrm{cm}^{-2}$, respectively. Backscattered protons were detected to monitor the target performance during each irradiation, using a Si surface barrier detector at an angle of $135^{\circ}$ with respected to the beam direction. In order to take into account the possible changes in the beam current, it was recorded with an integrator in time intervals of $1 \mathrm{~s}$. The applied current was between $80 \mathrm{nA}$ and $320 \mathrm{nA}$ depending on the thickness of the targets and beam energy.

After each irradiation, the target was removed from the target chamber and then transported to the $\gamma$ counting system in order to measure off-line the yield of the produced unstable isotopes, ${ }^{121} \mathrm{I}$ and ${ }^{120} \mathrm{I}$. The counting system was very similar to the one for our previous works on the $\alpha$ captures of ${ }^{106} \mathrm{Cd}[20]$ and ${ }^{112} \mathrm{Sn}$ [21]. The $\gamma$ - 
detection setup was composed of two Clover detectors placed face-to-face in close geometry, $4.9 \mathrm{~mm}$ gap between the Clovers. The whole setup is shielded against the room background with $5 \mathrm{~cm}$ of $\mathrm{Pb}$ and inner $\mathrm{Cu}$ lining of $3 \mathrm{~mm}$. The irradiated target was placed in a plexiglass holder, and then it was inserted between the Clovers, each of which had four individual crystals. Each crystal was utilized as a single detection unit operating in "direct" mode in order to reduce the pileup and summing losses. The energies of the crystals were recorded event by event together with the time of the event, and a pulser with a frequency $100 \mathrm{~Hz}$ was also applied to one of the Ge preamplifiers, so that the dead time was able to be reconstructed as a function of time.

\section{Analysis and results}

In such a close geometry, the detection efficiency is relatively high; the correction for coincidence summing effects could become important. The photopeak efficiency of the detection system was measured by the efficiency-ratio method, which is defined in Ref. [21]. The relative efficiencies were obtained with the uncalibrated ${ }^{152} \mathrm{Eu}$ source, and normalized to fit in with the absolute photopeak efficiency of calibrated ${ }^{137} \mathrm{Cs}$ source. The nearly $4 \pi$ detection geometry allowed the angular correlation to be neglected. The decay parameters used for the analysis and absolute photopeak efficiencies of the $\gamma$-transitions used for the products of ${ }^{120} \mathrm{Te}(\mathrm{p}, \gamma)$ and ${ }^{120} \mathrm{Te}(\mathrm{p}, \mathrm{n})$ reactions are given in Table I. In the case of ${ }^{120} \mathrm{Te}(\mathrm{p}, \mathrm{n})$, the product ${ }^{120} \mathrm{I}$ has ground and isomeric states, and their partial cross sections can be determined separately. The ${ }^{120} \mathrm{Te}(\mathrm{p}, \mathrm{n}){ }^{120} \mathrm{I}$ reaction cross section was determined by summing the partial cross sections of ${ }^{120} \mathrm{Te}(\mathrm{p}, \mathrm{n}){ }^{120 \mathrm{~g}} \mathrm{I}$ and ${ }^{120} \mathrm{Te}(\mathrm{p}, \mathrm{n}){ }^{120 \mathrm{~m}} \mathrm{I}$ reactions.

Table I. Decay parameters of the investigated reaction products [22] and measured photopeak efficiencies of the $\gamma$-transitions used for the analysis.

\begin{tabular}{|c||c||c||c|c||c|}
\hline Reactions & Product & Half-life (min) & $\mathbf{E}_{\boldsymbol{\gamma}}(\mathbf{k e V})$ & $\boldsymbol{\gamma}$-Intensity (\%) & \&ff. (\%) \\
\hline \hline${ }^{120} \mathrm{Te}(\mathrm{p}, \gamma)$ & ${ }^{121} \mathrm{I}$ & $127.2 \pm 0.6$ & 532.08 & $6.1 \pm 0.3$ & $12.6 \pm 0.3$ \\
\hline \multirow{2}{*}{${ }^{120} \mathrm{Te}(\mathrm{p}, \mathrm{n})$} & ${ }^{120 \mathrm{~g}} \mathrm{I}$ & $81.6 \pm 0.2$ & 1523.0 & $10.9 \pm 0.6$ & $5.3 \pm 0.1$ \\
\cline { 2 - 6 } & ${ }^{120 \mathrm{~m}} \mathrm{I}$ & $53 \pm 4$ & 654.5 & $2.1 \pm 0.7$ & $10.6 \pm 0.2$ \\
\hline
\end{tabular}

For the ${ }^{120} \mathrm{Te}(\mathrm{p}, \gamma){ }^{121} \mathrm{I}$ and ${ }^{120} \mathrm{Te}(\mathrm{p}, \mathrm{n}){ }^{120} \mathrm{I}$ reactions, the measured cross sections have been compared with the Hauser-Feschbach statistical model calculations obtained with the standard settings of the statistical model code NON-SMOKER [23] as seen in Figure 1 and Figure 2. The uncertainty in the results is governed by the following partial errors: the uncertainties in target thickness ( $9 \%$ ), counting statistics ( $0.2 \%$ to $11 \%$ ), detection efficiency (1.8 \% to $2.3 \%$ ), and decay parameters (0.2\% to $33 \%)$.

Although a good agreement is observed at energies up to $6.45 \mathrm{MeV}$ for the ${ }^{120} \mathrm{Te}(\mathrm{p}, \gamma){ }^{121} \mathrm{I}$ cross section values, the theoretical calculations deviate considerably at the higher energies from the measured data. The $(\mathrm{p}, \mathrm{n})$ channel opens around this energy (Q-value of 6.937 MeV corresponding to the threshold value of $6.451 \mathrm{MeV}$ ) and the model calculations for ${ }^{120} \mathrm{Te}(\mathrm{p}, \mathrm{n}){ }^{120} \mathrm{I}$ reaction underestimate measured values, see Figure 2 . The detailed final results will be published soon. 


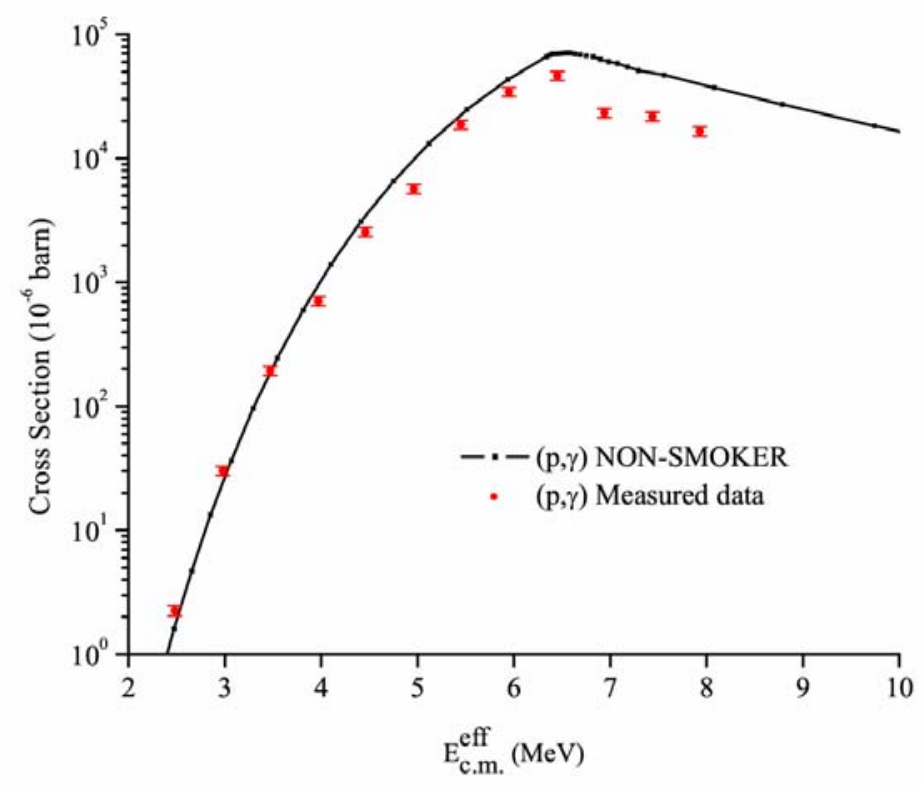

Figure 1. The measured cross sections for ${ }^{120} \mathrm{Te}(\mathrm{p}, \gamma)^{121} \mathrm{I}$ reaction in comparison with standard NON-SMOKER predictions [23].

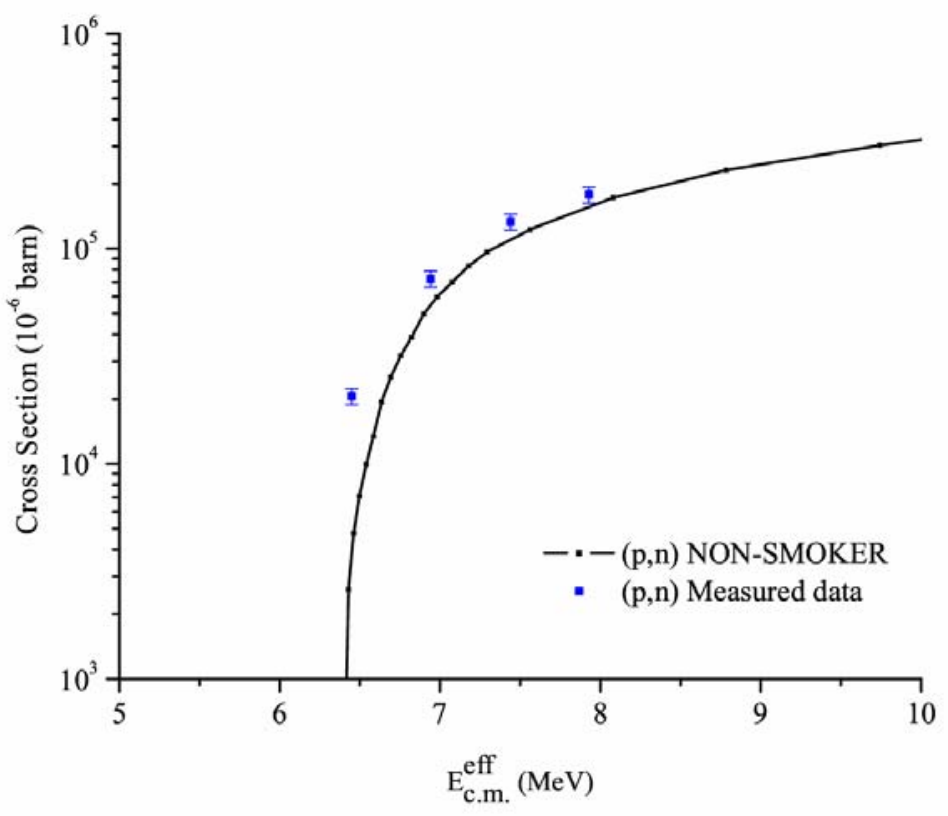

Figure 2. The measured cross sections for ${ }^{120} \mathrm{Te}(\mathrm{p}, \mathrm{n})^{120} \mathrm{I}$ reaction in comparison with standard NON-SMOKER predictions [23]. 


\section{Acknowledgements}

This work was supported by The Scientific and Technical Research Council of Turkey TUBITAK - Grant 108T508, the National Science Foundation NSF- Grant 0434844, the Joint Institute for Nuclear Astrophysics JINA (www.JINAweb.org) PHY02-16783, and The Hungarian Scientific Research Fund Programs OTKA (K68801, T49245).

\section{References}

[1] S. E. Woosley and W. M. Howard, Ap. J. Suppl. 36 (1978) 285.

[2] M. Arnould, S. Goriely, Phys. Rep. 384 (2003) 1.

[3] H. Utsunomiya et al. Nucl. Phys. A777 (2006) 459.

[4] K. Sonnabend et al. Phys. Rev. C 70 (2004) 035802.

[5] G. G. Kiss et al. Phys. Rev. C 76 (2007) 055807.

[6] Gy. Gyürky et al.Phys. Rev. C 68 (2003) 055803.

[7] Gy. Gyürky et al. Phys. Rev. C 64 (2001) 065803.

[8] S. Galanopoulos et al. Phys. Rev. C 67 (2003) 015801.

[9] P. Tsagari et al. Phys. Rev. C 70 (2004) 015802.

[10] C. E. Laird et al. Phys. Rev. C Phys. 35 (1987) 1265.

[11] S. Harissopulos et al. Phys. Rev. C 64 (2001) 055804.

[12] T. Sauter, F. Kappeler, Phys. Rev. C 55 (1997) 3127.

[13] F. R. Chloupek et al. Nucl. Phys. A652 (1999) 391.

[14] J. Bork et al. Phys. Rev. C 58 (1998) 524.

[15] N. Özkan et al. Nucl. Phys. A710 (2002) 469.

[16] Gy. Gyürky et al. J. Phys. G: Nucl. Part. Phys. 34 (2007) 817.

[17] M.A. Famiano et al. Nucl. Phys. A802 (2008) 26.

[18] N. Özkan et al. Eur. Phys. J. A Direct 27 (2006) 145.

[19] J. Greene et al. Nucl. Instr.: Meth. In Phys. Res. A 590 (2008) 76.

[20] Gy. Gyürky et al. Phys. Rev. C 74 (2006) 025805.

[21] N. Özkan et al. Phys. Rev. C 75 (2007) 025801.

[22] http://www.nndc.bnl.gov/nudat2/

[23] T. Rauscher and F. K. Thielemann, At. Data Nucl. Data Tables 79 (2001) 47. 